\title{
Overview of hydatidoses in albania and assessment of risk factors
}

\begin{abstract}
Introduction: Echinococcosis (Hydatidosis), this neglected iceberg, cosmopolitan zoonotic disease has a problematic character and affect more than one million people. The infection is transmitted through ingestion of parasite eggs or through direct contact with definitive animal hosts. ${ }^{1-4}$ The clinically diagnosed cases from imaging techniques, serologic assays and surgeon treatment, are only a small part visualized of the total number of infected people. The other invisible part of this disease is: health, social and economic consequences of the affected patients. Echinococcosis is often expensive and complicated to treat, and may require extensive surgery and/or prolonged drug therapy. ${ }^{5,6}$ Prevention programmes involve deworming of dogs, ${ }^{7}$ improved slaughterhouse hygiene, and public education campaigns. ${ }^{9-11}$
\end{abstract}

Aim: This retrospective study aims at displaying a detailed picture of Echinococcose situation assessing correlation with socio-economic conditions in Albania population. The epidemiologic data were collected from the individual case-report schedule for endemic control of zoonosis referring the person-place-time tirade. During five years period (20092013), were analyzed 658 hospitalized patients established in serological findings for antiEchinococcose antibodies, ultrasound, CT/RMI and surgery.

Conclusions: The available data according to this period performed that: Hydatidosis is widespread in different regions of Albania. The higher prevalence was presented in 2011 $(44.5 \%)$. The positivity of cases resulted at $31.4 \%$. The relative incidence of cases was $4.1 \%-4.7 \%$ (cases $/ 100.000$ people). Infestation rate related to gender was $61 \%$ female $/ 38 \%$ male. The new cases goes up to \pm 22 patients/year, comparing with earlier 2006 period $( \pm 14)$. About $85 \%$ of these patients underwent surgery as treatment. $11.1 \%$ of cases were recidivists. No reported data of lethality. Echinococcose may affect every age-group population.

This study identified several possible important risk factors: Socioeconomic status, environmental contamination, contaminated food, no vaccination of sheep, no deworming of dogs. It's very important the control of zoonotic diseases (Echinococcosis especially as a neglected diseases).

Keywords: zoonosis, echinococcosis/hydatidosis, definitive host, seroprevalence, incidence, age group
Volume 4 Issue 4 - 2017

Valbona Gjoni

Department of Control of Infectious Diseases, Albania

Correspondence: Valbona Gjoni, Department of Control of Infectious Diseases, Institute of Public Health, Tirana,Address: Rr. Aleksander Moisiu n.80,Tirana, Albania,

Email gjoni_valbona@yahoo.com

Received: February 02, 2016 | Published: March 30, 2017

\section{Introduction}

Hydatidosis or cystic Echinococcosis (CE) is a chronic neglected zoonotic disease of almost worldwide distribution caused by the cestode parasite Echinococcus granulosus whom, the predominant life cycle takes place in a synanthropic cycle with domestic dogs (Canis lupus familiaris) as definitive hosts and livestock animals as intermediate hosts. The disease represents a major public health problem and economic burden particularly. ${ }^{12-16}$ The aim of this review is to display a detailed picture of Echinococcose situation, assessing correlation of socio-economic associated factors with disease, in Albania population.

\section{Material and methods}

\section{Study area}

During five years period (2009-2013), were analyzed 658 hospitalized patients established in serological findings for antiEchinococcus antibodies, ultrasound, CT/RMI and surgery. The epidemiologic data were collected from the individual case-report schedule for endemic control of zoonosis referring the person-place- time triad and a specific database was created assessing the risk factors too. The residence of the patients that had moved during the time was chosen that they had lived during the last 10 years.

\section{Experimental}

A blood sample was taken from each participant by the intravenous blood. Specimens were centrifuged on the same day and the serum was separated and kept at $-20^{\circ} \mathrm{C}$ until further analysis. Human serum samples were all screened by a commercial enzyme-linked immunosorbent assay (ELISA) detecting IgG antibodies against $E$. granulosus antigens (Echinococcus IgG ELISA classic, Serion Immundiagnostica, Würzburg, Germany). Positive and indeterminate samples were retested, by additional serological tests ELISA at the suggested time.

\section{Results and discussion}

The available data according to this period performed that: Hydatidose is widespread in different regions of Albania (Tirana, Mati, Dibra,Mirdita, Përmeti). The ELISA screening revealed positive results in 207 out of 658 serum samples, resulting in a seropositivity 
of $31.5 \%$. The higher prevalence was presented in $2011(44.5 \%)$. The relative incidence of cases was $4.1 \%-4.7 \%$ (cases $/ 100.000$ people). Infestation rate related to gender was $61 \%$ female and $38 \%$ male. The new cases goes up to \pm 22 patients/year, comparing with earlier 2006 period $( \pm 14)$.

About $85 \%$ of these patients underwent surgery as treatment. $11.1 \%$ of cases were recidivists. No reported data of lethality. Echinococcose may affect every age-group population. The spread of cases according to residences in the years shows that $32.3 \%$ of the patients, are resident in village (rural) areas and $29.7 \%$ of them are resident in cities (urban) areas. The most affected people were farmers $(47.8 \%)$ and pet owners $(13 \%)$ compared to persons in other occupations $(1.9 \%)$. A higher seropositivity was found in people who reported high dog contact (49\%).

This study identified several possible important risk factors as environmental contamination, socioeconomic background linked with dog owners, food human and animal sources, knowledges about vaccination of sheep and deworming of dogs, allowing dogs to roam free, high dog-human contact. Epidemiologic data can provide important information to improve the control of the disease. It's very important the control of zoonotic diseases (Echinococcosis especially as a neglected disease).

\section{Conclusion}

Hydatidose is widespread in different regions of Albania (Tirana, Mati, Dibra,Mirdita, Përmeti). The new cases goes up to \pm 22 patients/year, comparing with earlier 2006 period $( \pm 14)$. No reported data of lethality. Echinococcose may affect every agegroup population. There are no significance change between patient's residence (32.3\% rural: $29.7 \%$ urban). Risk factors for human seropositivity were related to contact with deworming domestic dogs and their feces, and dogs that roam free. Echinococcose is a disease which may affect every age-group population and has affinity for the liver (41.5\%) and lung (21.7\%).

Although evidence is lacking, the exposures by uncontrolled food may be another risk for Echinococcosis. Epidemiologic data can provide important information to improve the control of the zoonotic diseases. There has never been a systematic review summarizing the significant determinants for Echinococcosis in humans and animals. The Ministry of Health and the Ministry of Food and Agriculture both, has to instigate control programs.

\section{Conflicts of Interest}

The authors declare no conflict of interest.

\section{Acknowledgments}

None.

\section{Funding}

None.

\section{References}

1. Eckert J, Conraths FJ, Tackmann K. Echinococcosis: an emerging or re-emerging Zoonosis. Int J Parasitol. 2000;30(12-13): 1283-1294.

2. Torgerson PR, Budke CM. Echinococcosis-an international public health challenge. Res Vet Sci. 2003;74(3):191-202.

3. Eckert J, Deplazes P. Biological, Epidemiological, and Clinical Aspects of Echinococcosis, a Zoonosis of Increasing Concern. Clin Microbiol Rev. 2004;17(1):107-135.

4. Parikh F. Echinococcosis cut to cure but what about control? $J$ Assoc Physicians India. 2012;60:9-10.

5. McManus DP, Gray DJ, Zhang W, et al. Diagnosis, treatment, and management of echinococcosis. BMJ. 2012;p. 344: e3866.

6. Arben P, Gentian V, Arben P, et al. A Neglected Zoonosis in Albania: why Echinococcosis is Becoming a Surgeon's Exclusivity? Mediterr J Hematol Infect Dis. 2014;6(1):e2014013.

7. Xhaxhiu D, Kusi I, Rapti D, et al. Principal intestinal parasites of dogs in Tirana, Albania. Parasitol Res. 2011;108(2):341-353.

8. Torgerson PR, Macpherson CN. The socioeconomic burden of parasitic zoonoses: global trends. Vet Parasitol. 2011;182(1):79-95.

9. Torgerson PR. One world health: Socioeconomic burden and parasitic disease control priorities. Vet Parasitol. 2013;195(3-4): 223-232.

10. Mathers C, Fat DM, Boerma J. The global burden of disease: 2004 update: World Health Organization. 2008.

11. Dorny P, Praet N, Deckers N, Gabriel S. Emerging food-borne parasites. Vet Parasitol. 2009;163(3):196-206.

12. World Health Organization. The control of neglected zoonotic diseases: A route to poverty alleviation. 2006; pp. 1-65.

13. World Health Organization. First WHO report on neglected tropical diseases: Working to overcome the global impact of neglected tropical diseases. 2010.

14. European Centre for Disease Prevention and Control. Annual epidemiological report on 2011 surveillance data and 2012 epidemic intelligence data 2013; p. 1-260.

15. EFSA and ECDC. The European Union Summary Report on Trends and Sources of Zoonoses, Zoonotic Agents and Food-borne Outbreaks in 2012. EFSA Journal. 2014;12(2):3547.

16. Grosso G, Gruttadauria S, Biondi A, et al. Worldwide epidemiology of liver hydatidosis including the Mediterranean area. World $J$ Gastroenterol. 2012;18(13):1425-1437. 